\title{
Controllability of structural brain networks and the waxing and waning of negative affect
}

\section{in daily life}

McGowan, A.L. ${ }^{1}$, Parkes, L. ${ }^{2}$, He, X. ${ }^{2,3}$, Stanoi, O. ${ }^{4}$, Kang, Y. ${ }^{1}$, Lomax, S. ${ }^{1}$, Jovanova, M., ${ }^{1}$ Mucha, P.J. ${ }^{5}$, Ochsner, K.N. ${ }^{4}$, Falk, E.B. ${ }^{1,6,7}$, Bassett, D.S. ${ }^{2,8,9,10,11,12}$, \& Lydon-Staley, D.M. ${ }^{1,2,13^{*}}$

${ }^{1}$ Annenberg School for Communication, University of Pennsylvania, Philadelphia, PA

${ }^{2}$ Department of Bioengineering, School of Engineering and Applied Science, University of Pennsylvania, Philadelphia, PA, USA

${ }^{3}$ Department of Psychology, School of Humanities and Social Sciences, University of Science and Technology of China, Hefei, P.R. China

${ }^{4}$ Department of Psychology, Columbia University, New York City, NY, USA

${ }^{5}$ Department of Mathematics and Applied Physical Sciences, University of North Carolina, Chapel Hill, North Carolina, USA

${ }^{6}$ Department of Psychology, University of Pennsylvania, Philadelphia, PA, USA

${ }^{7}$ Marketing Department, Wharton School, University of Pennsylvania, PA, USA

${ }^{8}$ Department of Physics \& Astronomy, College of Arts and Sciences, University of Pennsylvania, Philadelphia, PA, USA

${ }^{9}$ Department of Electrical \& Systems Engineering, School of Engineering and Applied Science, University of Pennsylvania, Philadelphia, PA, USA

${ }^{10}$ Department of Neurology, Perelman School of Medicine, University of Pennsylvania, Philadelphia, PA, USA

${ }^{11}$ Department of Psychiatry, Perelman School of Medicine, University of Pennsylvania, Philadelphia, PA, USA

${ }^{12}$ Santa Fe Institute, Santa Fe, NM, USA 
${ }^{13}$ Leonard Davis Institute of Health Economics, University of Pennsylvania, Philadelphia, PA, USA

*Corresponding author: David M. Lydon-Staley, 3620 Walnut St, Annenberg School for Communication, University of Pennsylvania, Philadelphia, PA 19104 USA. Email: david.lydonstaley@asc.upenn.edu

Keywords: affect variability, average controllability, depression, ecological momentary assessment, network control theory, cingulo-insular system

The most recent version of this manuscript has been accepted for publication at Biological Psychiatry: Global Open Science doi: https://doi.org/10.1016/j.bpsgos.2021.11.008 


\section{Acknowledgements}

Research was sponsored by the Army Research Office and was accomplished under Grant Number W911NF-18-1-0244. D.M.L. and A.L.M. acknowledge support from the National Institute on Drug Abuse (K01 DA047417) and the Brain \& Behavior Research Foundation. D.S.B. acknowledges support from the John D. and Catherine T. MacArthur Foundation, the Swartz Foundation, the Paul G. Allen Family Foundation, the Alfred P. Sloan Foundation and the NSF (PHY-1554488; IIS-1926757). D.S.B. and L. P. acknowledge support from the National Institute of Mental Health (R01MH113550), and L.P. acknowledges support from the 2020 NARSAD Young Investigator Grant from the Brain \& Behavior Research Foundation. The views and conclusions contained in this document are those of the authors and should not be interpreted as representing the official policies, either expressed or implied, of the Army Research Office or the U.S. Government. The U.S. Government is authorized to reproduce and distribute reprints for Government purposes notwithstanding any copyright notation herein.

\section{Citation Diversity Statement}

Recent work in several fields of science has identified a bias in citation practices such that papers from women and other minority scholars are under-cited relative to the number of such papers in the field (1-9). Here we sought to proactively consider choosing references that reflect the diversity of the field in thought, form of contribution, gender, race, ethnicity, and other factors. First, we obtained the predicted gender of the first and last author of each reference by using databases that store the probability of a first name being carried by a woman $(5,10)$. By this measure (and excluding self-citations to the first and last authors of our current paper), our references contain $23.73 \%$ woman(first)/woman(last), $16.95 \%$ man/woman, $15.25 \%$ 
woman/man, and $44.07 \% \mathrm{man} / \mathrm{man}$. This method is limited in that a) names, pronouns, and social media profiles used to construct the databases may not, in every case, be indicative of gender identity and b) it cannot account for intersex, non-binary, or transgender people. We look forward to future work that could help us to better understand how to support equitable practices in science.

\section{Financial Disclosures}

The authors have no biomedical financial interests or potential conflicts of interest. 


\begin{abstract}
Background: The waxing and waning of negative affect in daily life is normative, reflecting an adaptive capacity to respond flexibly to changing circumstances. However, understanding of the brain structure correlates of affective variability in naturalistic settings has been limited. Using network control theory, we examine facets of brain structure that may enable negative affect variability in daily life.
\end{abstract}

Methods: We use diffusion weighted imaging data from 95 young adults $\left(M_{\text {age }}=20.19\right.$ years, $S D_{\text {age }}=1.80 ; 56$ women) to construct structural connectivity networks that map white matter fiber connections between 200 cortical and 14 sub-cortical regions. We apply network control theory to these structural networks to estimate the degree to which each brain region's pattern of structural connectivity facilitates the spread of activity to other brain systems. We examine how the average controllability of functional brain systems relates to negative affect variability, computed by taking the standard deviation of negative affect self-reports collected via smartphone-based experience-sampling twice per day over 28 days as participants went about their daily lives.

Results: We find that high average controllability of the cingulo-insular system is associated with increased negative affect variability. We find that greater negative affect variability is related to the presence of more depressive symptoms, yet average controllability of the cinguloinsular system was not associated with depressive symptoms.

Conclusions: Our results highlight the role brain structure plays in affective dynamics as observed in the context of daily life, suggesting that average controllability of the cingulo-insular system promotes normative negative affect variability. 


\section{Controllability of structural brain networks and the waxing and waning of negative affect in daily life}

Our lives are given meaning and color by our capacity to experience dynamically evolving affective responses. The dynamic nature of these experiences $(11,12)$ has been documented in behavioral studies that densely sample self-reports of emotion in daily life (1315). These data show that affective variability — or shifts in levels of affective experience over time (16) - is normative and is indicative of a capacity to respond flexibly to changing conditions (16-19). Yet, there are important between-person differences in affect variability that are associated with psychological and physical health outcomes $(16,20)$. Between-person differences in affect variability likely stem from multiple sources and may be indicative of the individual, the context in which they are embedded, or the person-context system as a whole (21).

Here, we focus on the association between brain structure and negative affect variability. The brain can be conceived of as a network of neuronal ensembles or regions (nodes) interlinked by anatomical wires (edges) in a complex and patterned architecture. This connective structure supports the dynamics of neural activity as the brain transitions through functional brain states, traversing a path in a dynamic state-space landscape (Gu et al., 2018; Figure 1). The trajectory of these brain state transitions is modulated by both external and internal perturbations (23). The ease with which these internal perturbations - driven by activity in individual brain regions modulate the brain's trajectory depends on the strength and pattern of structural connections associated with that region (24). Network control theory is a branch of physical and engineering sciences that can be used to quantify the ease with which particular brain regions can modulate the brain's trajectory $(25,26)$. Network control theory models each brain region's activity as a time-dependent internal state that is predicted from a combination of three factors: (i) its 
previous state, (ii) whole-brain structural connectivity, and (iii) external inputs. After linearizing the system's dynamics, average controllability quantifies a brain region's capacity to distribute activity through the brain, via both direct and indirect connections, to guide changes in brain state. Average controllability complements conventional graph-theoretic metrics of structural brain organization, such as strength and centrality metrics that describe brain structure (27-29), by modeling how brain regions with particular patterns of connections with regions throughout the rest of the brain can facilitate particular dynamics in brain function.

Networks with high average controllability are more influential in the control of network dynamics, exhibiting greater capacity of driving the system into different states with little effort (i.e., input energy). Some individuals may have structural brain network architectures that more easily facilitate changes in brain state trajectories than others (i.e., high average controllability), and consequently, they may show greater negative affect variability. We hypothesize that individuals with functional brain systems encompassing cingulate and fronto-insular regions that can more readily drive the brain into different states will exhibit greater negative affect variability. Our hypothesis stems from the fact that the cingulo-insular functional system facilitates access to cognitive control resources (e.g., attention, working memory) that coordinate behavioral responses appropriate to meet the demands of situations (e.g., studying; 34-36). This functional system facilitates access to cognitive control by engaging the frontoparietal system while suppressing default mode system activity $(33,34)$. The cingulo-insular system's facilitation of flexible behavior may also stem from engagement with functional systems beyond the frontoparietal and default mode systems, with functional Magnetic Resonance Imaging studies indicating that nodes in this system have a unique spatiotemporal dynamic profile, showing substantial time-varying functional interactions with other functional systems $(35,36)$. Notably, 
between-person differences in the extent to which the cingulo-insular system interacts with other functional brain systems is positively correlated with cognitive flexibility $(35,36)$.

Given the cingulo-insular system's role in recruiting other brain systems to facilitate changes in behavior (32), we tested the hypothesis that individuals with brains exhibiting a pattern of structural connectivity that facilitates the spread of activity from this system to other systems of the brain and with the ability to drive the brain into different states (i.e., high average controllability), would also have high negative affect variability.

\section{Methods and Materials}

We used data from the Social and Health Impact of Network Effects (SHINE) study, a larger study designed to provide insight into health behaviors and social interactions among young adults. All research was conducted in accordance with the Institutional Review Board (IRB) at the University of Pennsylvania and Columbia University, in addition to the Army Research Office. All data and code used in the manuscript are available at http://osf.io/gkahy/. The source code for the average controllability calculation is available at http://github.com/nangongwubu/Network-Controllability-Diagnostics.

\section{Participants and Procedure}

Recruitment materials advertised a study titled "Social Health Impact of Network Effects Study (SHINE)" to undergraduate students who were members of on-campus social groups across two universities, University of Pennsylvania and Columbia University. The study was advertised through flyers, university websites, and email communication. To reach campus groups, researchers contacted group leaders as points of contact and further employed a snowball sampling approach, such that participating students could share recruitment information with their peers who were members of on-campus social clubs or sports teams. For the current report, 
912 individuals were invited to participate in the study (see Supplemental Figure 1 for a CONSORT flow diagram of enrollment and retention through the study periods). A subset of 661 individuals (72.48\%) of invited participants consented and agreed to take part in the study. These participants completed an online survey assessing fMRI eligibility and an hour-long baseline survey.

Following the baseline survey, participants meeting fMRI inclusion criteria and agreeing to participate in the next part of the study $(n=112)$ were randomized into three conditions as part of a larger investigation unrelated to the current report: control $(n=39)$, mindfulness $(n=38)$, and perspective-taking $(n=35)$. Participants attended a laboratory session that included surveys, an MRI session, and instructions for an ecological momentary assessment (EMA) and intervention (EMI) protocol designed to reduce alcohol use (findings are robust to controlling for intervention condition and, as such, we present the most parsimonious models without condition throughout this paper). The day following the laboratory session, participants began a 28-day ecological momentary assessment protocol. The EMA and EMI was completed by 112 participants from 10 groups in the following conditions: control $(n=39)$, mindfulness $(n=38)$, and perspective-taking $(n=35)$; however, final analyses were conducted on 95 participants following exclusion of participants who failed to complete the EMA component due to accidentally deleting the smartphone application from their phones $(n=5)$ or who were scanned with incorrect fMRI parameters $(n=12)$. Participants also completed 6-month and 12-month follow-up online surveys not presented here. Participants received up to $\$ 135$ for participating in the current study. Participants received a \$20 Amazon gift card for completing the online baseline survey, $\$ 50$ cash for completing the laboratory session, and a $\$ 55$ Amazon gift card for answering at least $70 \%$ of ecological momentary assessments, and an additional $\$ 10$ gift card if more than $80 \%$ of group 
members completed the online baseline survey. We focus on the baseline survey, the MRI session, and the ecological momentary assessment and refer readers to http://osf.io/gkahy/ for greater detail about the rest of the protocol.

The participants reported on in this manuscript comprise 95 young adults aged 18 to 28 years old $(M=20.19, S D=1.80,56$ women $)$. Participants identified as Asian $(30.5 \%)$; Black or African American (2.1\%); Latino/a (5.3\%); white (52.6\%); and as multiple categories: white, American Indian or Alaska Native (2.1\%); white, Asian (3.2\%); white, Asian, Native Hawaiian or other Pacific Islander (1.1\%); and white, Latino/a (3.2\%). Data collection began in February 2019 and ended in April 2020.

MRI data acquisition, preprocessing, and modeling. Imaging data were acquired on 3Tesla Siemens Trio scanners equipped with a 64-channel head coil. The DWI data were preprocessed and reconstructed through QSIprep v 0.8.0 (37). Briefly, the data was first denoised and bias corrected, and then underwent susceptibility distortion correction, motion and eddy current correction via FSL 6.0, and coregistered to T1 space. We also warped both the Schaefer atlas (38) and the Harvard Oxford subcortical atlas (39) into individual T1 space to subdivide the brain into 200 cortical and 14 subcortical regions. Then, the preprocessed DWI data was reconstructed using generalized Q-sampling Imaging (40) in DSI-Studio (http://dsi-

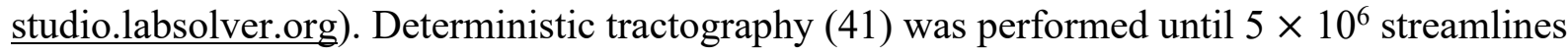
were reconstructed, yielding individual structural networks with brain regions as nodes and the number of streamlines connecting each brain region pair as weighted edges. Preprocessing was performed using QSIPrep 0.8.0, which is based on Nipype 1.4.2 (49,50; RRID:SCR_002502). See supplemental methods for greater detail of anatomical and diffusion data preprocessing. 
Ecological momentary assessment. On the day after the laboratory session, participants began a 28-day ecological momentary assessment period. Each day, participants answered two signal-contingent surveys per day. A morning survey was sent at 8:00 AM and an evening survey was sent at 6:00 PM. The surveys assessed affect, alcohol consumption, and a range of other variables not reported on in this manuscript (see http://osf.io/gkahy/ for codebook). Participants completed on average $52.44 \pm 6.56$ reports $($ minimum $=18$, maximum $=56)$. The number of responses was unrelated to negative affect or negative affect variability $(r$ 's $\leq 0.03, p$ 's $\geq 0.65$ [95\% CI: -0.24 to 0.23$])$.

\section{Measures}

We used participants' reports of demographic information from the baseline surveys, their ratings of negative affect during the 28-day experience-sampling period, and diffusion weighted imaging (DWI) to create structural brain networks.

Negative affect. Negative affect was measured every morning and evening in response to the question "How negative do you feel right now?" on a scale of 1 (not at all) to 100 (extremely) in increments of 1 . Out of a possible total of 5376 negative affect reports, 4982 $(92.7 \%)$ were available. Participants completed 18 to 56 affect reports $(M=52.44, S D=6.56)$ across the experience-sampling period. Average negative affect was calculated using the intraindividual mean across each participant's negative affect report timeseries $(M=37.38, S D=$ 14.29). Negative affect variability was calculated using the intraindividual standard deviation across each participant's negative affect reports timeseries $(M=17.88, S D=6.44)$.

Depression. Depression was measured using the 10-item version of the Center for Epidemiological Studies-Depression Scale (CESD-10; Radloff, 1977). All items included four response categories indicating the frequency of depressive symptoms during the past week on a 
four-point scale of 0 (rarely or none of the time, less than 1 day), 1 (some or a little of the time, 1-2 days), 2 (occasionally or a moderate amount of the time, 3-4 days), or 3 (most or all of the time, 5-7 days). The scoring of positive items is reversed and the possible range of scores is 0 to 30, with higher scores indicating the presence of more depressive symptoms. Scores $\geq 10$ indicate significant depressive symptoms (45). Participants' depressive symptoms ranged from 0 to $22($ Median $=9, S D=5)$. Using the $\geq 10$ cut-off score, 47 participants were classified as not having significant depressive symptoms and 45 participants were classified as having significant depressive symptoms $(n=3$ participants failed to complete the CESD portion of the baseline survey).

Average controllability. From the DWI data, we constructed anatomical brain networks by subdividing the brain into 214 regions using the Schaefer atlas for 200 cortical regions and the Harvard Oxford atlas for 14 subcortical regions. In these anatomical connectivity matrices, brain regions are defined as nodes, and a link between two nodes represents the number of streamlines connecting them, normalized for density (46). We drew on Network Control Theory to assess the extent to which large-scale brain networks exert control over other large-scale brain networks. Controllability of a dynamical system describes the possibility of driving the current state of a system to a desired target state via external control input (47). Here, we focus on average controllability, which quantifies each region's capacity to leverage the brain's underlying structural connectivity to distribute activity throughout the brain to guide changes between easily reachable states (25). Networks with high average controllability are more influential in the control of network dynamics, driving the system into different states with little effort (i.e., input energy). The relationship of the mathematical formulation of network control to brain networks is discussed in more detail in Gu et al. (25). To ensure system stability, each participant's 
structural connectivity matrix was normalized by dividing each element by the largest absolute eigenvalue of the matrix plus one (26). Following normalization, average controllability was calculated for each node. Next, rank-based inverse normal transformations were applied to each node across participants to ensure normality (48). Finally, we calculated the mean average controllability over nodes within each of 17 functional brain systems $(38,49)$. These systemaveraged estimates of average controllability were taken into subsequent analyses of betweenperson differences (see below for further details).

\section{Statistical Analysis}

We tested the extent to which negative affect variability was associated with average controllability of 17 functional brain systems (49) using 17 separate multilevel models, one for each system. We used multilevel models to account for the nested nature of the data (95 participants nested in 10 groups). We included average negative affect, total brain volume, and in-scanner motion as covariates. In all models, we specified a random intercept for group (as participants were nested in social groups). All analyses used the nlme package in $\mathrm{R}(50)$. We controlled for multiple comparisons given our examination of 17 systems using the BenjaminiHochberg (51) false discovery rate control.

\section{Results}

Following well-established methods $(13,16,52)$, we operationalized negative affect variability by taking the intraindividual standard deviation of up to 56 reports ( 2 per day for 28 days) of current negative affect. Participants with higher negative affect variability showed a greater range in their negative affect across time relative to participants with lower negative affect variability (Figure 2). 


\section{Average controllability of the cingulo-insular system is positively associated with negative}

\section{affect variability}

We provide descriptive statistics and correlations of the variables used in the analyses in Supplemental Table 1. We found that average controllability of the cingulo-insular system (labeled Salience/Ventral Attention A in the Yeo et al., 2011 atlas (49); nodes included in this

functional system are listed in Supplemental Table 2 and associations between these nodes and neurosynth meta-analysis maps are listed in Supplemental Table 3) was positively associated with greater negative affect variability $\left(b=9.57, p=0.01, p_{F D R}=0.03\right.$, Cohen's $d=0.57$; see Supplemental Table 4, Figure 3). Notably, this association was observed when controlling for covariates, including total brain volume, in-scanner motion, and average negative affect. We included average negative affect to ensure the association was specific to variability and not confounded with average negative affect (13), given that higher average self-reported negative affect was positively associated with negative affect variability $(b=0.13, p=0.004$, Cohen's $d=$ 0.65, see Supplemental Table 4). We provide results of the multilevel models examining associations between average controllability and negative affect variability in the 16 remaining functional brain networks in Supplemental Table 6. Notably, no associations were significant following false discovery rate control and effect sizes were small (Cohen's $d \leq 0.29, p$ 's $\geq 0.20$, $p_{F D R}{ }^{\prime} \geq 0.47$; see Table 1$)$.

We additionally examined the extent to which our results were specific to average controllability by computing the average strength, clustering coefficient, betweenness centrality, and closeness centrality (four commonly used network indices) of the 17 functional brain systems. No associations between negative affect variability and these additional network measures reached statistical significance following false discovery rate control ( $p$ 's $\geq 0.04$, 
$p_{F D R}{ }^{\prime} \geq 0.10$; see Supplemental Analyses). We direct readers to Supplemental Table 9 for correlations among these network metrics within each of the 17 subsystems.

We additionally conducted an analysis at the node rather than the system level to test which specific brain regions were associated with negative affect variability. After controlling for multiple comparisons, we observed a significant association between average controllability of the RH_SalVentAttnA_Ins_2 node (a node in the cingulo-insular system) and negative affect variability $\left(b=1.91, p=0.003, p_{F D R}=0.008\right.$, Cohen's $d=0.69$; see Table 2$)$. No other nodes survived false discovery rate control $\left(p_{F D R}\right.$ 's $\left.\geq 0.54\right)$, but of the top 5 nodes with the strongest effect sizes, 4 of them belonged to the cingulo-insular system (supplemental node-level analyses are contained in the supplemental Excel file).

To determine the specificity of the association between average controllability of the cingulo-insular system and negative affect variability, we ran multilevel models to assess the association between average controllability and average negative affect in addition to other emotion dynamic metrics. We found no significant associations between average controllability and average negative affect $(p ' s \geq 0.11)$, negative affect instability $\left(p=0.03, p_{F D R}=0.06\right.$; see Supplemental Table 7), or negative affect inertia $\left(p=0.21, p_{F D R}=0.64\right.$; see Supplemental Table 8). Supplemental Table 10 shows correlations among negative affect variability, instability, and inertia (see Supplemental Analyses for further details regarding calculations for negative affect instability and negative affect inertia).

Average controllability of the cingulo-insular system promotes normative negative affect variability

In follow-up analyses, we tested the extent to which the observed association between average controllability of the cingulo-insular network reflects normative variation in negative 
affect variability versus variability that may place individuals at risk for psychopathology. In line with previous work (53-55), a multilevel Poisson regression revealed that greater negative affect variability related to the presence of more depressive symptoms as measured by the Center for Epidemiological Studies-Depression Scale (CESD; $b=0.02, p=0.001, d=0.63$, Supplemental Figure 2). However, there was little evidence that average controllability of the cingulo-insular system was statistically significantly associated with depressive symptoms $(b=0.35, p=0.11, d$ $=0.15)$, indicating that negative affect variability was unlikely to mediate the association between controllability of this functional system and depressive systems. Finally, when depressive symptoms were included as a covariate in the regression model testing the association between controllability of the cingulo-insular system and negative affect variability, average controllability of the cingulo-insular system remained associated with negative affect variability ( $b=8.42, p=0.03$, Cohen's $d=0.50$; see Supplemental Table 5).

\section{Discussion}

We sought to explore the structural organization of brain systems underlying betweenperson differences in the extent to which negative affect fluctuates during daily life. We tested the hypothesis that individuals with cingulo-insular systems that have greater ability to facilitate the spread of activity to other brain systems (i.e., high average controllability) will show greater negative affect variability in daily life. In line with this hypothesis, we found that average controllability of the cingulo-insular system (Salience/Ventral Attention A in the Yeo 2011 atlas (49)) is positively associated with greater negative affect variability. Supplementary analyses demonstrate that other network metrics (i.e., strength, centrality, clustering coefficient) that describe brain structure organization were not associated with negative affect variability. This suggests that network control theory, and other model-based approaches that offer insight into 
the dynamics made possible by between-person differences in brain structure, may be particularly sensitive for understanding how brain structure organization influences changes in affective states.

The association between average controllability and negative affect variability was specific to the cingulo-insular system. This suggests that this functional system may serve as a key control point in structural brain networks subserving affect variability in daily life. Such an interpretation is consistent with the cingulo-insular system's unique role as a system that facilitates behavioral responses to detected events by signaling the engagement and suppression of other brain systems $(30,31)$. Due to having structural brain network architectures that facilitate the spread of activity from the cingulo-insular network to other systems, individuals with cingulo-insular systems with high average controllability may be more capable of changing their behavior when salient events are detected, which in turn may manifest as greater negative affect variability when these changes in behavior are recorded over extended periods of time. Future experimental work is necessary to test the extent to which average controllability relates to behavior as related to detecting salient events.

An additional finding of interest is that average controllability of the salience system was unrelated to daily-life average negative affect. This result indicates that having a cingulo-insular system with a pattern of structural connectivity that facilitates the spread of activity to other brain systems is implicated in experiencing greater variability in changing affective states rather than simply experiencing an overall greater intensity of negative affect. Further, average controllability of the cingulo-insular system was associated with the spread or deviation from one's average level of affect over time but not with other temporally dynamic processes (e.g., affect instability and affect inertia). This specificity further speaks to the cingulo-insular 
system's role in affect variability. Notably, the ability of the cingulo-insular network to engage with other systems of the brain to promote behavior change is often highlighted as a boon to promote cognitive, affective, and behavioral flexibility $(35,36)$. The current findings suggest that brain network structures that facilitate an especially strong influence of the cingulo-insular system on other brain systems promotes flexibility in affect.

Importantly, follow-up analyses confirm previous work indicating that excessive negative affect variability is associated with greater symptoms of depression (55). However, there was little evidence that this excessive variability, better conceptualized as affective lability (56) and emotion dysregulation, was associated with average controllability of the cingulo-insular system. Instead, average controllability of the cingulo-insular system was associated with normative variation in negative affect variability, in line with the system's role in cognitive and affective flexibility (36).

\section{Study limitations and future directions}

Despite the methodological strengths of the present investigation, the findings are not without limitations. First, an interval-contingent ecological momentary assessment protocol was used whereby participants responded to prompts at the same time every day. This approach likely supported the high response rate ( $92.7 \%$ of reports were completed), but it may have also led participants to change their behaviors in anticipation of assessments in a way that may be mitigated with signal contingent or variable time-based approaches. Second, the study sample exhibited low levels of depression. Thus, findings may not generalize to samples experiencing more severe levels of depression. Although our findings demonstrate no gender-related differences in the relationship between negative affect variability and average controllability, prior work has observed significant sex differences in functional connectivity in the cingulo- 
insular network (57) and in tractography when mapping the connectome (58). Future investigations should examine how biological sex assigned at birth relates to average controllability of the cingulo-insular system. Finally, we focused on average controllability, which estimates the extent to which individual brain regions distribute activity to other brain regions across the whole brain. Given that engagement of cingulo-insular regions with regions of the frontoparietal and default mode systems are specifically highlighted in theories of cognitive and behavioral flexibility $(48,49)$, future network control applications may, with suitable analytic frameworks (59), be capable of determining the extent to which findings are driven by a wholebrain spatio-temporal diversity of activity of cingulo-insular regions $(35,36)$ relative to engagement with the default mode and fronto-parietal systems specifically.

In sum, we find that between-person differences in the ease with which the cinguloinsular system can drive the brain into different states are associated with between-person differences in negative affect variability as observed in daily life. These findings provide insight into the role of brain structure in everyday affective experiences and provide additional support for the cingulo-insular system as a key functional brain system involved in affective dynamics. 


\section{References}

1. Bertolero MA, Dworkin JD, David SU, Lloreda CL, Srivastava P, Stiso J, et al. (2020): Racial and ethnic imbalance in neuroscience reference lists and intersections with gender. bioRxiv.

2. Caplar N, Tacchella S, Birrer S (2017): Quantitative evaluation of gender bias in astronomical publications from citation counts. Nature Astronomy 1: 1-5.

3. Chakravartty P, Kuo R, Grubbs V, McIlwain C (2018): \# CommunicationSoWhite. Journal of Communication 68: 254-266.

4. Dion ML, Sumner JL, Mitchell SM (2018): Gendered citation patterns across political science and social science methodology fields. Political Analysis 26: 312-327.

5. Dworkin JD, Linn KA, Teich EG, Zurn P, Shinohara RT, Bassett DS (2020): The extent and drivers of gender imbalance in neuroscience reference lists. arXiv preprint $\operatorname{arXiv:200101002.}$

6. Fulvio JM, Akinnola I, Postle BR (2020): Gender (im) balance in citation practices in cognitive neuroscience. Journal of Cognitive Neuroscience 1-5.

7. Maliniak D, Powers R, Walter BF (2013): The gender citation gap in international relations. International Organization 67: 889-922.

8. Mitchell SM, Lange S, Brus H (2013): Gendered citation patterns in international relations journals. International Studies Perspectives 14: 485-492.

9. Wang X, Dworkin J, Zhou D, Stiso J, Falk E, Zurn P, et al. (2020): Gendered Citation Practices in the Field of Communication. 
10. Zhou D, Cornblath EJ, Stiso J, Teich EG, Dworkin JD, Blevins AS, Bassett DS (2020):

Gender diversity statement and code notebook v1. 0. 2020. URL https://doi org/105281/zenodo 3672110.

11. Hollenstein T (2015): This time, it's real: Affective flexibility, time scales, feedback loops, and the regulation of emotion. Emotion Review 7: 308-315.

12. Kuppens P, Verduyn P (2017): Emotion dynamics. Current Opinion in Psychology 17: 2226.

13. Eid M, Diener E (1999): Intraindividual variability in affect: Reliability, validity, and personality correlates. Journal of Personality and Social Psychology 76: 662.

14. Frijda NH (1986): The Emotions. Cambridge University Press.

15. Liu H, Xie QW, Lou VW (2019): Everyday social interactions and intra-individual variability in affect: A systematic review and meta-analysis of ecological momentary assessment studies. Motivation and Emotion 43: 339-353.

16. Röcke C, Brose A (2013): Intraindividual variability and stability of affect and well-being. GeroPsych.

17. Brose A, Schmiedek F, Koval P, Kuppens P (2015): Emotional inertia contributes to depressive symptoms beyond perseverative thinking. Cognition and Emotion 29: 527538.

18. Kuppens P, Allen NB, Sheeber LB (2010): Emotional inertia and psychological maladjustment. Psychological science 21: 984-991.

19. Merz EL, Roesch SC (2011): Modeling trait and state variation using multilevel factor analysis with PANAS daily diary data. Journal of Research in Personality 45: 2-9. 
20. Hardy J, Segerstrom SC (2017): Intra-individual variability and psychological flexibility: Affect and health in a National US sample. Journal of Research in Personality 69: 13-21.

21. Koffer R, Ram N (2015): Intraindividual variability. The Encyclopedia of Adulthood and Aging 1-7.

22. Gu S, Cieslak M, Baird B, Muldoon SF, Grafton ST, Pasqualetti F, Bassett DS (2018): The energy landscape of neurophysiological activity implicit in brain network structure. Scientific reports $8: 1-15$.

23. Bassett DS, Khambhati AN (2017): A network engineering perspective on probing and perturbing cognition with neurofeedback. Annals of the New York Academy of Sciences 1396: 126.

24. Wu-Yan E, Betzel RF, Tang E, Gu S, Pasqualetti F, Bassett DS (2018): Benchmarking measures of network controllability on canonical graph models. Journal of Nonlinear Science 1-39.

25. Gu S, Pasqualetti F, Cieslak M, Telesford QK, Yu AB, Kahn AE, et al. (2015): Controllability of structural brain networks [no. 1]. Nature Communications 6: 8414.

26. Karrer TM, Kim JZ, Stiso J, Kahn AE, Pasqualetti F, Habel U, Bassett DS (2020): A practical guide to methodological considerations in the controllability of structural brain networks. Journal of neural engineering 17: 026031.

27. Fornito A, Zalesky A, Breakspear M (2015): The connectomics of brain disorders. Nature Reviews Neuroscience 16: 159-172.

28. Oldham S, Fulcher B, Parkes L, Arnatkevičiūtė A, Suo C, Fornito A (2019): Consistency and differences between centrality measures across distinct classes of networks. PloS one 14: e0220061. 
29. Dennis EL, Thompson PM (2013): Typical and atypical brain development: a review of neuroimaging studies. Dialogues in clinical neuroscience 15: 359.

30. Menon V (2015): Salience Network: Brain Mapping: An Encyclopedic Reference. Elsevier.

31. Uddin LQ (2015): Salience processing and insular cortical function and dysfunction. Nature reviews neuroscience 16: 55-61.

32. Uddin LQ (2021): Cognitive and behavioural flexibility: neural mechanisms and clinical considerations. Nature Reviews Neuroscience 22: 167-179.

33. Bonnelle V, Ham TE, Leech R, Kinnunen KM, Mehta MA, Greenwood RJ, Sharp DJ (2012): Salience network integrity predicts default mode network function after traumatic brain injury. Proceedings of the National Academy of Sciences 109: 4690-4695.

34. Sridharan D, Levitin DJ, Menon V (2008): A critical role for the right fronto-insular cortex in switching between central-executive and default-mode networks. Proceedings of the National Academy of Sciences 105: 12569-12574.

35. Chen T, Cai W, Ryali S, Supekar K, Menon V (2016): Distinct global brain dynamics and spatiotemporal organization of the salience network. PLoS biology 14: e1002469.

36. Lydon-Staley DM, Kuehner C, Zamoscik V, Huffziger S, Kirsch P, Bassett DS (2019): Repetitive negative thinking in daily life and functional connectivity among default mode, fronto-parietal, and salience networks. Translational psychiatry 9: 1-12.

37. Cieslak M, Cook PA, He X, Yeh F-C, Dhollander T, Adebimpe A, et al. (2020): QSIPrep: An integrative platform for preprocessing and reconstructing diffusion MRI. bioRxiv.

38. Schaefer A, Kong R, Gordon EM, Laumann TO, Zuo X-N, Holmes AJ, et al. (2018): Localglobal parcellation of the human cerebral cortex from intrinsic functional connectivity MRI. Cerebral cortex 28: 3095-3114. 
39. Smith SM, Jenkinson M, Woolrich MW, Beckmann CF, Behrens TE, Johansen-Berg H, et al. (2004): Advances in functional and structural MR image analysis and implementation as FSL. Neuroimage 23: S208-S219.

40. Yeh F-C, Wedeen VJ, Tseng W-YI (2010): Generalized q-sampling imaging. IEEE transactions on medical imaging 29: 1626-1635.

41. Yeh F-C, Verstynen TD, Wang Y, Fernández-Miranda JC, Tseng W-YI (2013):

Deterministic diffusion fiber tracking improved by quantitative anisotropy. PloS one 8: e80713.

42. Gorgolewski KJ, Burns CD, Madison C, Clark D, Halchenko YO, Waskom ML, Ghosh SS (2011): Nipype: a flexible, lightweight and extensible neuroimaging data processing framework in python. Frontiers in neuroinformatics 5: 13.

43. Gorgolewski KJ, Esteban O, Markiewicz CJ, Ziegler E, Ellis DG, Notter MP, et al. (2018): Nipype. Software, Zenodo.

44. Radloff LS (1977): The CES-D scale: A self-report depression scale for research in the general population. Applied Psychological Measurement 1: 385-401.

45. Zhang W, O’Brien N, Forrest JI, Salters KA, Patterson TL, Montaner JSG, et al. (2012): Validating a Shortened Depression Scale (10 Item CES-D) among HIV-Positive People in British Columbia, Canada. PLoS One 7: e40793.

46. Sotiropoulos SN, Zalesky A (2019): Building connectomes using diffusion MRI: why, how and but. NMR in Biomedicine 32: e3752.

47. Tang E, Bassett DS (2018): Colloquium: Control of dynamics in brain networks. Reviews of modern physics 90: 031003. 
48. McCaw ZR, Lane JM, Saxena R, Redline S, Lin X (2020): Operating characteristics of the rank-based inverse normal transformation for quantitative trait analysis in genome-wide association studies. Biometrics 76: 1262-1272.

49. Yeo BTT, Krienen FM, Sepulcre J, Sabuncu MR, Lashkari D, Hollinshead M, et al. (2011):

The organization of the human cerebral cortex estimated by intrinsic functional connectivity. J Neurophysiol 106: 1125-1165.

50. Pinheiro J (2009): nlme: linear and nonlinear mixed effects models. R package version 3.196. http://cran r-project org/web/packages/nlme/.

51. Benjamini Y, Hochberg Y (1995): Controlling the false discovery rate: a practical and powerful approach to multiple testing. Journal of the Royal statistical society: series B (Methodological) 57: 289-300.

52. Brose A, Schmiedek F, Gerstorf D, Voelkle MC (2020): The measurement of within-person affect variation. Emotion 20: 677.

53. Chan DK, Zhang X, Fung HH, Hagger MS (2016): Affect, affective variability, and physical health: results from a population-based investigation in China. International journal of behavioral medicine 23: 438-446.

54. Kashdan TB, Rottenberg J (2010): Psychological flexibility as a fundamental aspect of health. Clinical Psychology Review 30: 865-878.

55. Waugh CE, Thompson RJ, Gotlib IH (2011): Flexible emotional responsiveness in trait resilience. Emotion 11: 1059.

56. Harvey PD, Greenberg BR, Serper MR (1989): The affective lability scales: development, reliability, and validity. Journal of clinical psychology 45: 786-793. 
57. Conrin SD, Zhan L, Morrissey ZD, Xing M, Forbes A, Maki P, et al. (2018): From Default Mode Network to the Basal Configuration: Sex Differences in the Resting-State Brain Connectivity as a Function of Age and Their Clinical Correlates. Frontiers in Psychiatry 9: 365 .

58. Ingalhalikar M, Smith A, Parker D, Satterthwaite TD, Elliott MA, Ruparel K, et al. (2014): Sex differences in the structural connectome of the human brain. Proceedings of the National Academy of Sciences 111: 823-828.

59. Stiso J, Khambhati AN, Menara T, Kahn AE, Stein JM, Das SR, et al. (2019): White matter network architecture guides direct electrical stimulation through optimal state transitions. Cell reports 28: 2554-2566.

60. Manoliu A, Meng C, Brandl F, Doll A, Tahmasian M, Scherr M, et al. (2014): Insular dysfunction within the salience network is associated with severity of symptoms and aberrant inter-network connectivity in major depressive disorder. Frontiers in human neuroscience 7: 930.

61. Parkes L, Moore TM, Calkins ME, Cieslak M, Roalf DR, Wolf DH, et al. (2021): Network controllability in transmodal cortex predicts positive psychosis spectrum symptoms. Biological Psychiatry. https://doi.org/10.1016/j.biopsych.2021.03.016

62. Tang E, Giusti C, Baum GL, Gu S, Pollock E, Kahn AE, et al. (2017): Developmental increases in white matter network controllability support a growing diversity of brain dynamics. Nature communications 8: 1-16. 
Table 1. Effect sizes (Cohen's $d$ ) for associations between average controllability and negative affect variability in the 17 functional brain networks in descending order.

\begin{tabular}{lcc}
\hline \multicolumn{1}{c}{ Network } & Cohen's $\boldsymbol{d}$ & $\boldsymbol{p}_{\text {FDR }}$ \\
\hline Salience/Ventral Attention A (cingulo-insular) & 0.57 & 0.03 \\
Control A & 0.29 & 0.47 \\
Default A & 0.27 & 0.53 \\
Default B & 0.25 & 0.58 \\
Limbic A & 0.23 & 0.68 \\
Visual Peripheral (Visual B) & 0.22 & 0.68 \\
Default C & 0.21 & 0.72 \\
Control C & 0.19 & 0.77 \\
Dorsal Attention A & 0.19 & 0.77 \\
Salience/Ventral Attention B & 0.17 & 0.85 \\
Control B & 0.17 & 0.85 \\
Somatomotor B & 0.14 & 0.95 \\
Dorsal Attention B & 0.11 & 0.95 \\
Visual Central (Visual A) & 0.10 & 0.99 \\
Limbic B & -0.04 & 0.99 \\
Temporal Parietal & -0.10 & 0.99 \\
Somatomotor B & -0.14 & 0.99 \\
\hline Note. Reported $p$-values $\left(p_{F D n)}\right.$ are following false discovery rate control $(51)$
\end{tabular}

Note. Reported $p$-values $\left(p_{F D R}\right)$ are following false discovery rate control (51). 
Table 2. Results of multilevel model examining associations between average controllability with negative affect variability in nodes within the cingulo-insular system.

\begin{tabular}{|c|c|c|c|c|c|c|}
\hline \multirow[b]{2}{*}{ Effect } & \multirow[b]{2}{*}{ Estimate } & \multirow[b]{2}{*}{$\begin{array}{l}\text { Standard } \\
\text { error }\end{array}$} & \multirow[b]{2}{*}{$p$} & \multirow[b]{2}{*}{$d$} & \multicolumn{2}{|c|}{ Confidence interval } \\
\hline & & & & & Lower & Upper \\
\hline \multicolumn{7}{|l|}{ RH_SalVentAttnA_Ins_2 } \\
\hline \multicolumn{7}{|l|}{ Fixed effects } \\
\hline Intercept & $13.40 * * *$ & 1.77 & $<0.001$ & & 9.88 & 16.91 \\
\hline Average controllability & $1.91^{* *}$ & 0.61 & 0.003 & 0.70 & 0.68 & 3.13 \\
\hline Negative Affect & $0.12 * *$ & 0.04 & 0.005 & 0.64 & 0.04 & 0.21 \\
\hline Total brain volume & 0.17 & 0.61 & 0.78 & 0.06 & -1.04 & 1.39 \\
\hline In-scanner motion & 0.12 & 0.73 & 0.87 & 0.04 & -1.33 & 1.57 \\
\hline \multicolumn{7}{|l|}{ Random effects } \\
\hline Intercept & \multicolumn{2}{|c|}{1.65} & & & & \\
\hline Residual & \multicolumn{2}{|c|}{34.04} & & & & \\
\hline
\end{tabular}

Note. 95 participants nested in 10 groups; ${ }^{* * *} p<0.001 ; * * p \leq 0.01$. Total brain volume and inscanner motion were sample-mean centered; average controllability was rank-based inverse normal transformed. RH_SalVentAttnA_Ins_2 was the only node surviving false discovery rate control. See supplemental Table 2 for coordinates. 


\section{Figure Captions}

Figure 1. Construction of average controllability indices. A. Participants $(n=95)$ underwent diffusion weighted imaging. B. The resulting data underwent tractography to map white matter fiber streamline connections between 214 cortical and subcortical regions. $\mathbf{C}$. The resulting structural brain networks consist of nodes (brain regions) connected by edges, links between nodes representing the number of streamlines connecting them, normalized for density (46). D. Structural brain networks are analyzed in a network control framework $(25,60,61)$ to compute the structural support that network offers for moving the brain to easy-to-reach states following control input. Figures adapted from Parkes et al. (61) and Tang et al. (62).

Figure 2. Between-person differences in negative affect variability. A. Participants provided up to 56 reports (two reports per day for 28 days) of their negative affect on their smartphones as they went about their daily lives in an ecological momentary assessment protocol. The time series of negative affect reports of two participants is shown in panels $\mathbf{B}$ and $\mathbf{C}$. Both participants exhibit similar mean $(M)$ values of negative affect across the 56 reports, as indicated by the black dashed line. However, the participant in panel $\mathbf{C}$ shows greater variability in their negative affect around their mean affect, relative to the participant in panel $\mathbf{B}$ as highlighted in grey. This greater negative affect variability is captured by the intraindividual standard deviation of the negative affect time series $(S D)$.

Figure 3. Greater average controllability of the cingulo-insular system is associated with greater negative affect variability. A. Multilevel models indicate that the strongest association, indicated by Cohen's $d$, between negative affect variability and average controllability was observed in the cingulo-insular system labelled as Salience/Ventral Attention A in 
(49). The association between average controllability and negative affect variability was only significant in the cingulo-insular system. B. Participants with higher average controllability values (x-axis; average of rank-based inverse normal transformed within each network within each individual) exhibited higher negative affect variability (y-axis) in their daily lives. Note that the association between average controllability and negative affect variability remains significant when the potential outlier (not depicted in the figure) is removed. 
Figure 1.

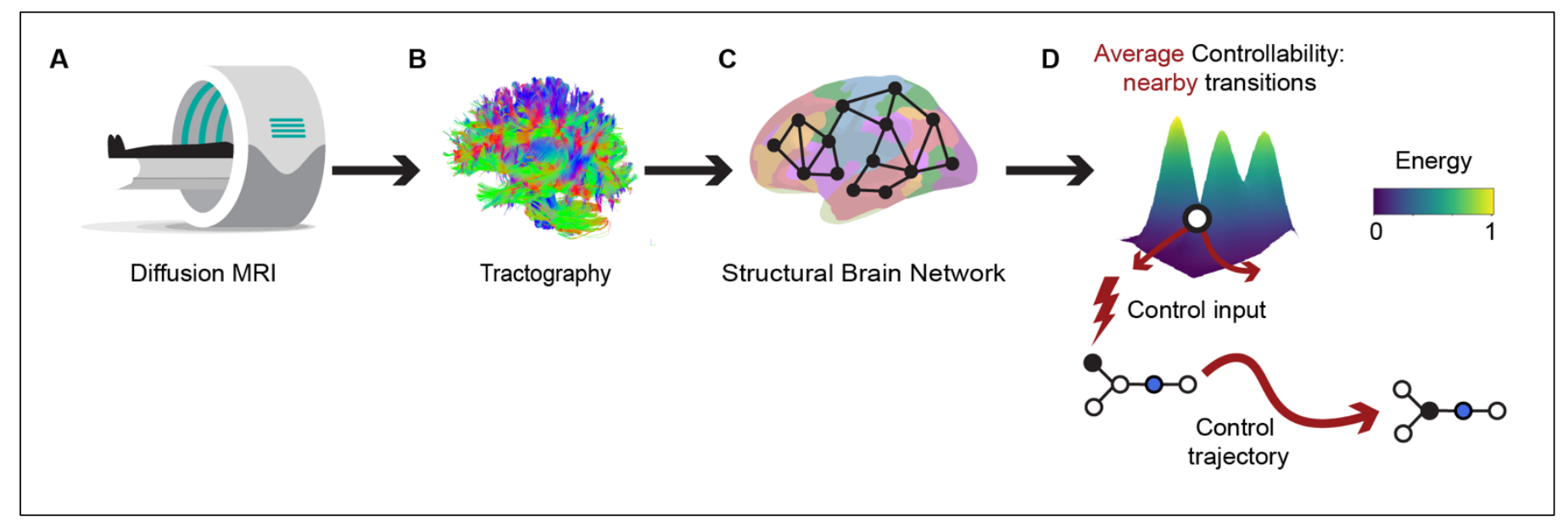


Figure 2.

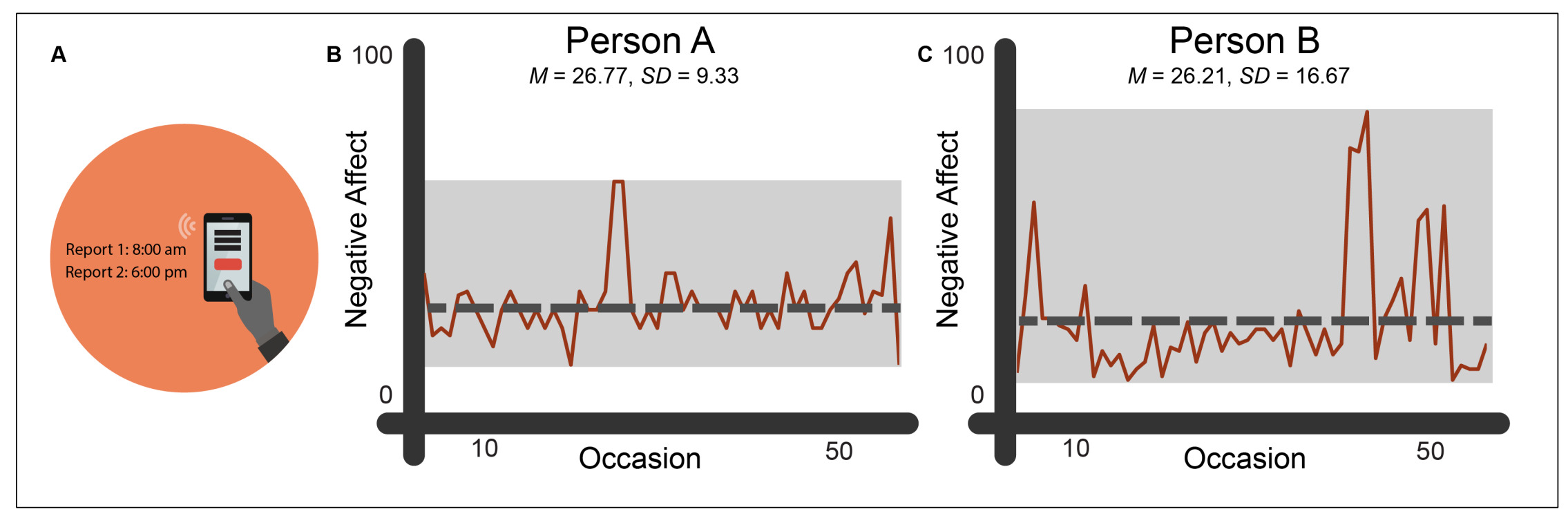


Figure 3.

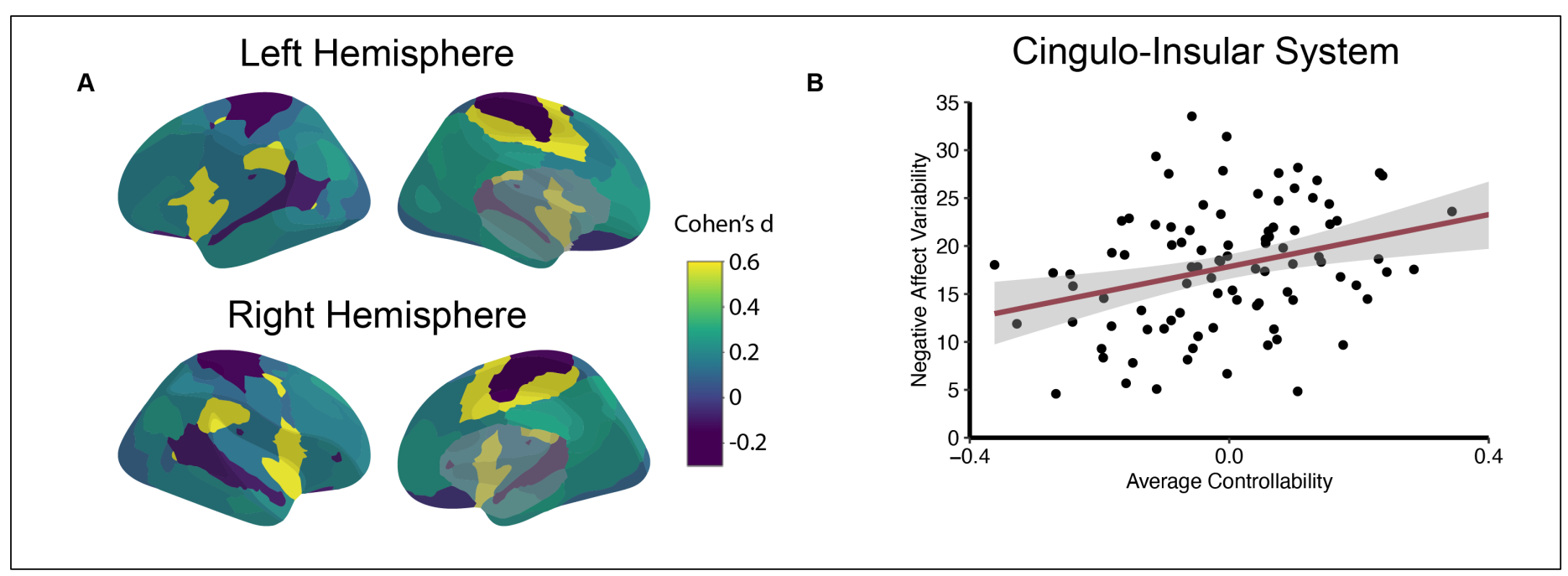

